\title{
Electric Field Induced Changes in Surface Bonding: Integrating First Principles and Atom Probe Tomography
}

\author{
Claudia Loyola, Joaquin Peralta, Scott Broderick and Krishna Rajan \\ Department of Materials Science and Engineering/Iowa State University, Ames, IA, USA
}

In this work we analyze the field evaporation process by studying the rupture phenomena of the bond between the ad-atoms and surface by linking atom probe tomography (APT) experimental data and first principles calculated ad-atom charge distributions. Evaporation field is a phenomenon that occurs when surface atoms start to evaporate under a strong electric field [1], which is utilized in APT by applying a voltage over a specimen tip. Those atoms are detected [2], and based on this information the specimen tip is reconstructed. We investigate this evaporation process on an $\mathrm{Al}_{3} \mathrm{Sc}$ surface under the presence of a strong electric field by using Density functional theory (DFT) with Quantum-ESPRESSO (QE) software [3]. A detailed description of the variation of the bonding between the surface and the ad-atoms is evaluated for a wide range of electric fields incorporated on the simulation cell.

The surface contains a slab of 80 atoms of $\mathrm{Al}_{3} \mathrm{Sc}$ in the $<111>$ direction along the $\mathrm{z}$-axis. The calculations were performed for four different configurations of ad-atoms on the surface: Al, Sc, Al-Al and Al-Sc. In these calculations we employed a plane wave basis set, generalized gradient approximation (GGA) and pseudopotentials. The electric field was incorporated as an additional term in the Hamiltonian and was applied to all simulations using the dipole correction [4], which is implemented in the QE package. The advantage of this method is that it avoids problems generated by the periodicity of the system in the z-axis within the vacuum space.

The maximum electric field applied to the system varies for each ad-atom and dimer on the surface and ranges from 0 to 25, 29, 32 and $36 \mathrm{~V} / \mathrm{nm}$ for $\mathrm{Al}-\mathrm{Al}, \mathrm{Al}, \mathrm{Sc}$, and $\mathrm{Al}-\mathrm{Sc}$, respectively [5]. The electric field is increased by $1 \mathrm{~V} / \mathrm{nm}$ each step, giving us a total of 126 simulations. The distance of the ad-atom to the surface is not modified during the simulation. To determine the charge density of the bond between the ad-atoms and the surface, three different charge densities have been calculated. The first is an initial charge density, $\rho_{0}$ that corresponds to the charge density of the full system; the second is the charge density associated to the surface without the ad-atoms, $\rho_{1}$; and the third one is the charge density of only the ad-atoms, $\rho_{2}$. The final charge density of the bond was determined using $\rho_{\text {bond }}=\rho_{0}-\rho_{1}-\rho_{2}$. The boding charge density for an Al-Al dimer on the surface with two different electric fields is shown in figure 1 .

Based on the calculated 3D charge density between the ad-atoms and the surface, we select some planes of interest to analyze the bonding change under the presence of a strong electric field. Figure 2 shows the bonding of the Al-Al ad-atoms and the surface at two different electric fields. The first plane, $\mathrm{P}^{1} \mathrm{AlAl}$, corresponds to the plane that intersects a dimer and a $\mathrm{Sc}$ atom from the surface $\left(\mathrm{Sc}_{\mathrm{s}}\right)$, and from which we find that they share charge density. The second plane, $\mathrm{P}^{2} \mathrm{AlAl}$, is defined by one $\mathrm{Al}$ atom from the dimer and two atoms from the surface, $\mathrm{Sc}_{\mathrm{s}}$ and $\mathrm{Al}_{\mathrm{s}}$. The bond of these atoms corresponds to a significant charge density. This work describes an exhaustive analysis of the charge bonding of the adatoms and the surface with the presence of a strong electric field. Variations of the bonding are clearly observed. This work uses charge population analysis to relate the charge around the evaporated atoms to the detected ions in Atom Probe Tomography [6]. 
[1] R. G. Forbes, Applied Surface Science 87/88 (1995), p. 1-11.

[2] M. K. Miller, and R. G. Forbes, Materials Characterization 60 (2009), p. 461-469.

[3] P. Giannozzi et al, Journal of Physics: Condensed Matter 21 (2009), p. 395502.

[4] L. Bengsston, Physical Review B 59 (1999), p. 12301-12304.

[5] J. Peralta et al, Ultramicroscopy (In Press).

[6] Support comes from Air Force Office of Scientific Research grants: FA9550-10-1-0256, FA955011-1-0158 and FA9550-12-0496; NSF grants: ARI Program CMMI-09-389018 and PHY CDI-0941576; and Defense Advanced Research Projects Agency grant N66001-10-1-4004
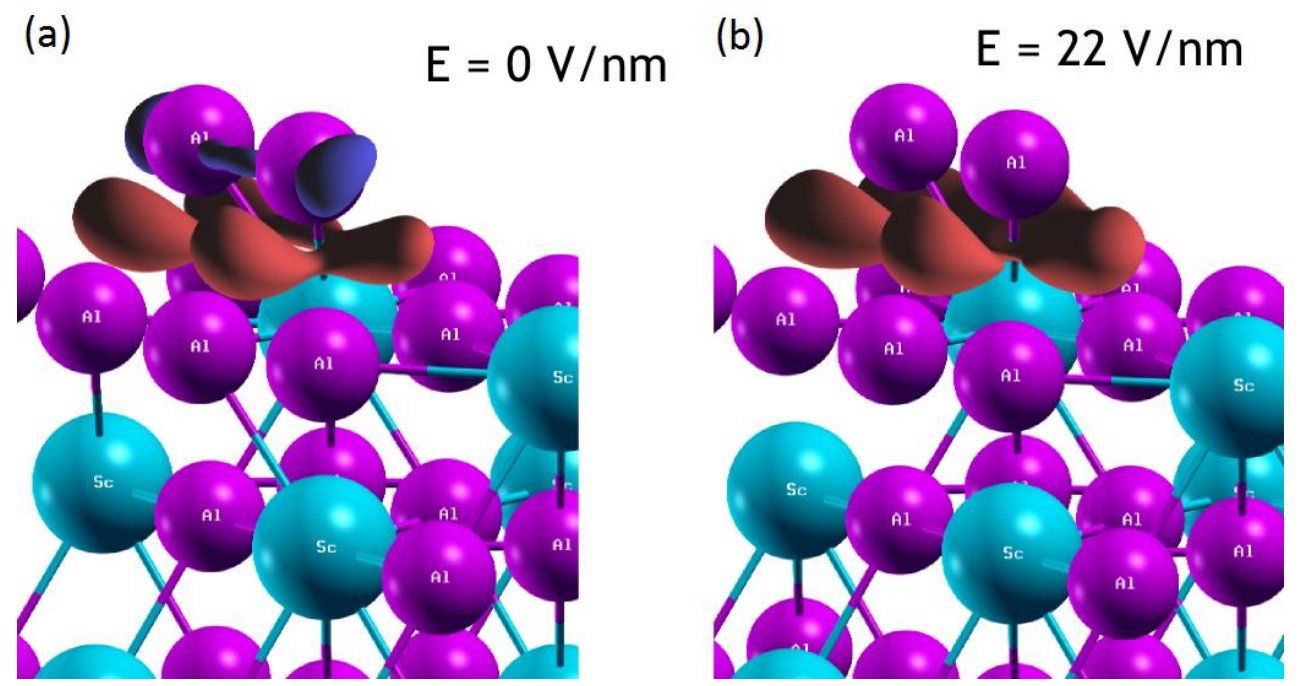

Figure 1. Change in the charge distribution Al-Al dimer on the surface, as a function of electric field.

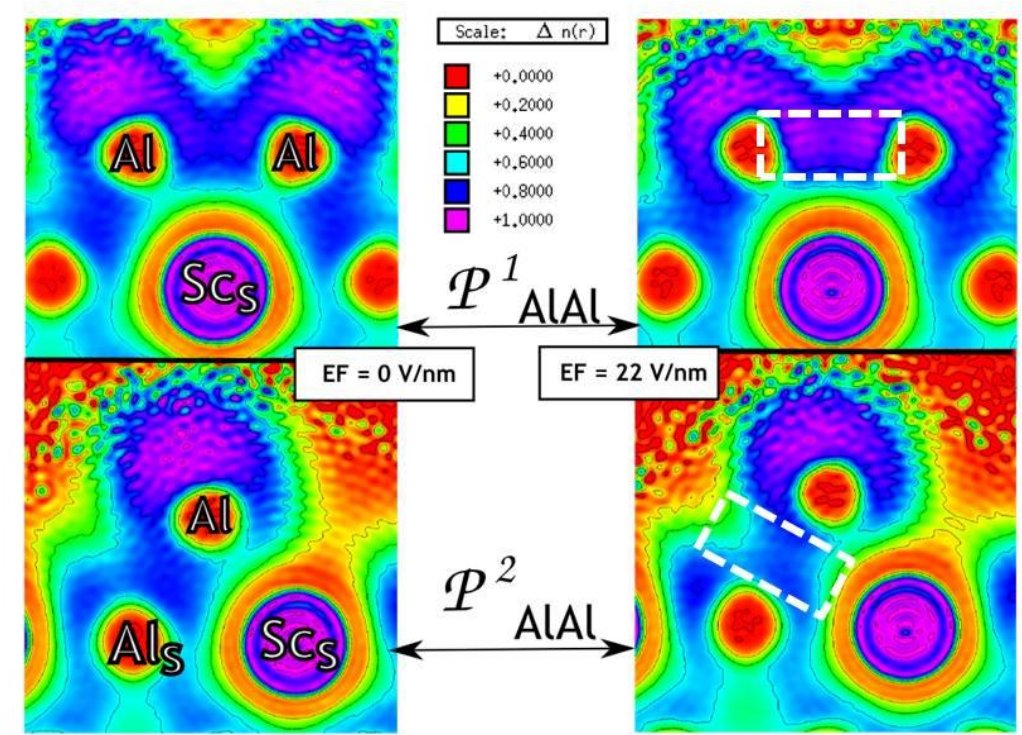

Figure 2. Charge density in two planes of interest. These show the changes in bonding between Al-Al ad-atoms and the surface due to changing electric field (boxed region). 